\title{
Potencial de ingresos pasivos para los distribuidores en empresas de mercadeo multinivel con un plan de compensación binario $^{1}$
}

Recibido:

Junio 16 de 2012

Aceptado:

Noviembre 10 de 2012

\section{Resumen}

En este trabajo se desarrolla una simulación de un sistema de mercadeo multinivel con un sistema de pago binario, con el fin de determinar la existencia de ingresos pasivos para sus distribuidores ya que este tipo de negocios generan altas expectativas de ingresos y que, por su baja inversión, puede llegar a personas de todas las clases sociales. El mercadeo multinivel es una forma de comercializar productos a través de estructuras sociales que ha tenido un gran crecimiento en Colombia en los últimos años y que ha captado el interés de las grandes empresas. Para la simulación, se generó inicialmente una red multinivel de 5000 distribuidores y después se adaptó a un sistema de pago binario para simular los ingresos de cada uno de ellos. Los algoritmos se desarrollaron en el software Matlab y los gráficos de las redes en el software Pajek. Los resultados de la simulación arrojaron que el $80 \%$ de las ventas las generan el $4 \%$ de los distribuidores con muy pocas ventas directas (4\%) y que solo el 1,2\% de los distribuidores generan un ingreso pasivo. Finalmente, se considera importante utilizar este tipo de herramientas dada la dificultad de probar estas formas de comercialización en sistemas sociales reales.

Palabras clave: ingreso pasivo, mercadeo multinivel, sistema de pago binario.

\section{Abstract}

This paper develops a simulation of a multilevel marketing system with a binary payment system, in order to determine the existence of passive income for its distributors because that kind of businesses generate high expectations of incomes and that, for its low investment, can reach people from all social classes. The multilevel marketing is a way to market products through social structures that have had a great growth in Colombia in recent years and has attracted the interest of big business. For the simulation, initially generated a multilevel network of 5000 distributors and then adapted to a binary payment system to simulate
${ }^{3}$ Universidad Nacional de Colombia - Sede Manizales, Facultad de Administración, Departamento de Informática y Computación, Manizales, 170003 - Colombia.

Correo electrónico: gaosorioz@unal.edu.co 
income each of them. The algorithms were developed in the Matlab software and the networks graphs in Pajek software. The simulation results showed that $80 \%$ of sales are generated for $4 \%$ of the distributors with few direct sales (4\%) and only $1.2 \%$ of distributors generate a passive income. Finally, it is important to use these tools because of the difficulty of proving these forms of marketing in real social systems.

Keywords: passive income, multilevel marketing, binary payment system.

\section{Introducción}

$E^{1}$ mercadeo multinivel (MLM) es una forma de distribución y comercialización de productos o servicios de consumo masivo (Vander Nat y Keep, 2002), directamente desde el fabricante al consumidor final a través de una red de personas que generan un ingreso por referir dichos productos o servicios (Coughlan y Grayson,1998). El fabricante estimula al consumidor para que refiera sus productos a su círculo social y establezca una venta efectiva (Kim et al., 2006). A pesar de su crecimiento, algunos autores han cuestionado este tipo de comercialización de productos, tanto por su parecido a esquemas piramidales como por las altas expectativas que se generan entre sus distribuidores por la posibilidad de obtener grandes ingresos o ingresos pasivos (Legara et al., 2008; Martinez, 2007; Vander Nat y Keep, 2002). Este último, es un ingreso recibido de forma regular con poco esfuerzo necesario para mantenerlo (Kiyosaki y Sharon, 2000).

Según la IRS (The American Internal Revenue Service) el ingreso pasivo solamente proviene de dos fuentes: actividades de arrendamientos o actividades de empresa o comerciales en la que no se participa materialmente. Algunos ejemplos son: la renta de una propiedad, la pensión y ganancias de un negocio que no requiere la participación directa del propietario o comerciante. Para el presente trabajo se utilizó el último ejemplo, donde un distribuidor genera ingresos constantes sin la necesidad de realizar un alto número de ventas directas. Por lo tanto, se tuvo en cuenta dos consideraciones: el porcentaje de distribuidores que ganaban el $80 \%$ de las comisiones y la cantidad de ventas directas que realizaban para generar dichas comisiones.

Por otro lado, algunas investigaciones que se han realizado son las de Coughlan y Grayson (1998) que simularon el crecimiento de una red de acuerdo a las diferentes actividades que hace el distribuidor. Legara et al. (2008) utilizaron el modelo del Mundo Pequeño de redes sociales y el modelado basado en agentes para realizar una simulación de ingresos de los distribuidores, dando como resultado que el crecimiento de ingresos no es ilimitado y van disminuyendo por la finitud de los posibles conocidos de cada miembro de la red. Cruz (2009) utilizó la teoría de redes complejas para generar una red multinivel y el enfoque de Dinámica de Sistemas para estudiar el proceso de difusión de productos, concluyendo que este modelo de comercialización sí funciona a diferencia de otros modelos ilegales como los piramidales. Pedrood et al. (2008) realizó una función de costos para simular el crecimiento de una red de mercadeo multinivel con 
un sistema de pago binario y determinó que existiría una mayor penetración en el mercado si los distribuidores recibieran menos comisión en un menor tiempo, en vez de una retribución alta en un periodo más amplio.

En este trabajo se realizó la simulación de los ingresos de los distribuidores teniéndose en cuenta las principales formas de pago que existen en el mercadeo multinivel (Dumitra, 2009), que son: escalonado o por ruptura, uninivel, unigen y plan binario. El plan binario es el de mayor popularidad (Pedrood et al., 2008), por esta razón se escogió para llevar a cabo la simulación.

El plan binario tiene una anchura limitada a 2 personas y una profundidad ilimitada. Para cobrar se tiene que compensar los dos lados y siempre existirá un lado débil que será el que defina las comisiones, junto con el tiempo establecido por la compañía. Un ejemplo se encuentra en la Figura 1, cuyo proceso se explica en la sección 2.2

Figura 1. Ciclos y pasos en un sistema de pago binario

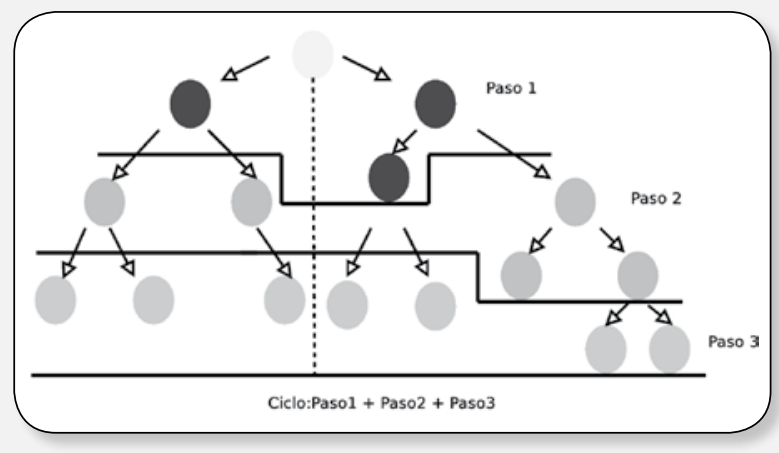

A nivel global, el mercadeo multinivel y la venta directa han tenido un desarrollo importante. Para diciembre 31 de 2010 las ventas al por menor fueron de 132.222 millones de dólares con un total de 87.681.031 vendedores (Federación Mundial de Asociaciones de Venta Directa - WFDSA, 2010). El mercadeo multinivel ha sido adoptado por empresas de distintos sectores en todo el mundo. Algunos ejemplos son: Amway, Excel, Forever and Living Products, Herbalife, Mary Kay, Nokken, NuSkin Enterprises, Shaklee y Primerica, entre otras. Sin embargo, ha estado bajo una estricta vigilancia debido a su similitud con estructuras Poisson o esquemas piramidales (Vander Nat y Keep, 2002). Adicional a esto, se han presentado otros cuestionamientos como: su capacidad de comercializar productos o servicios por medio de estructuras sociales (Taylor, 2002) y su potencial de generar ingresos ilimitados (Legara et al., 2008).

Aunque el mercadeo multinivel está muy alejado de un sistema piramidal común, ya que utiliza el consumo de productos para realizar los pagos a su estructura de distribuidores y no por vinculaciones de personas, se cuestiona el hecho que el origen de los pagos a los distribuidores que se encuentran en la cima, sea exclusivamente o en un porcentaje muy alto, por las compras de personas que están haciendo el negocio y no por la venta a consumidores finales. Por lo anterior, Vander Nat y Keep (2002) proponen que al menos el $70 \%$ del origen de los ingresos generados por los distribuidores sean por ventas a clientes finales que no desarrollen el negocio, para validar que el sistema de pago no presente un comportamiento piramidal.

Además, el mercadeo multinivel ha sido cuestionado por las altas expectativas de ingresos que se generan. Este tipo de negocios presenta una llamativo "enriquézcase rápido" o "gane un ingreso pasivo o pensión" y como su inversión es pequeña puede llegar a muchas personas de niveles de ingresos bajos, haciéndolas soñar con grandes fortunas. Por lo tanto, es necesario analizar la posibilidad de que se generen ganancias de este tipo (Legara et al. 2008). ISSN 0122-820X

Existe una gran dificultad para realizar estudios al mercadeo multinivel porque no utilizan la publicidad tradicional sino las estructuras sociales para determinar sus ventas. Por ejemplo, cuando se utiliza la publicidad tradicional se pueden hacer proyecciones de ventas y estimar el comportamiento del mercado, lo que no ocurre cuando se utiliza la venta multinivel. Algunos enfoques que se han empleado para realizar estos análisis han 
sido el modelo de Red de Mundo Pequeño (Pedrood et al. 2008) y de redes complejas (Cruz, 2009).

Lo que se pretendió con esta investigación fue analizar la posibilidad de que un negocio de mercadeo multinivel genere ingresos pasivos a sus distribuidores, haciendo uso de una simulación. Para esta tarea, se generó una red de 5000 distribuidores y después se aplicó un sistema de pago binario para determinar los ingresos de cada uno. Partiendo del algoritmo propuesto por Cruz y Olaya (2008), se simuló una red de mercadeo multinivel y a la red resultante, se le aplicó un plan de compensación binario para simular los ingresos de los distribuidores.

\section{Marco Experimental}

\subsection{Simulación de la red multinivel}

Para realizar la simulación de la red de mercadeo multinivel, se partió del algoritmo desarrollado por Cruz y Olaya (2008) el cual fue implementado en el software de Dinámica de Sistemas iThink®. Este modelo estaba limitado a nodos de grado 10, lo que significa que el número máximo de nuevos distribuidores vinculados por un distribuidor era de 10. Para poder ampliar este número, a uno ilimitado, se implementó el mismo algoritmo en Matlab® 2010a. El diagrama de flujo de dicho algoritmo, se puede observar en la Figura 2.

Figura 2. Diagrama de flujo del algoritmo a partir de

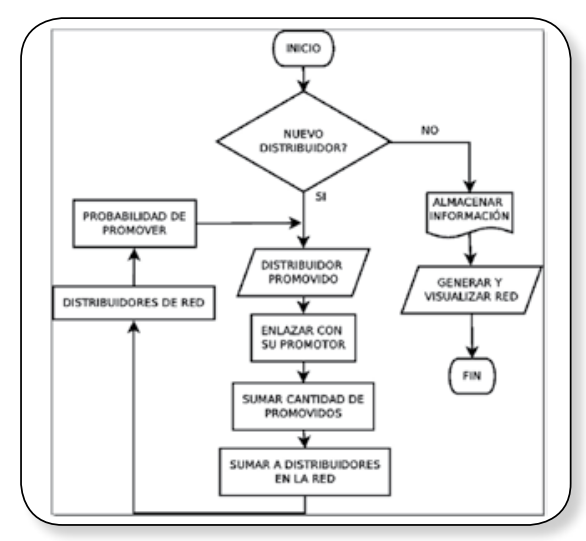

Se inicia con dos nodos conectados, cuando aparece un nuevo nodo (distribuidor) se halla la probabilidad de grado de cada uno de los nodos existentes y se suman, luego se genera un número aleatorio y se compara con las probabilidades de grado calculadas anteriormente. De acuerdo a esta comparación se enlaza el nuevo nodo con su promotor y se actualiza el grado de los nodos en la red. Se halla la nueva probabilidad de grado y se suma a la anterior para determinar la probabilidad de grado acumulada. Se genera el número aleatorio y se realiza el mismo proceso hasta que el número total de nodos sea igual al número ingresado por el usuario.

En la Figura 3, se muestra la evolución inicial de 5.000 nodos para $10,50,100$ y 200 nodos. Siempre se inicia con dos nodos para poder generar la red y se utilizó el software Pajek ${ }^{\circledR} 2.04$ para visualizar las redes.

Figura 3. Evolución inicial de una red de 5000 distribuidores

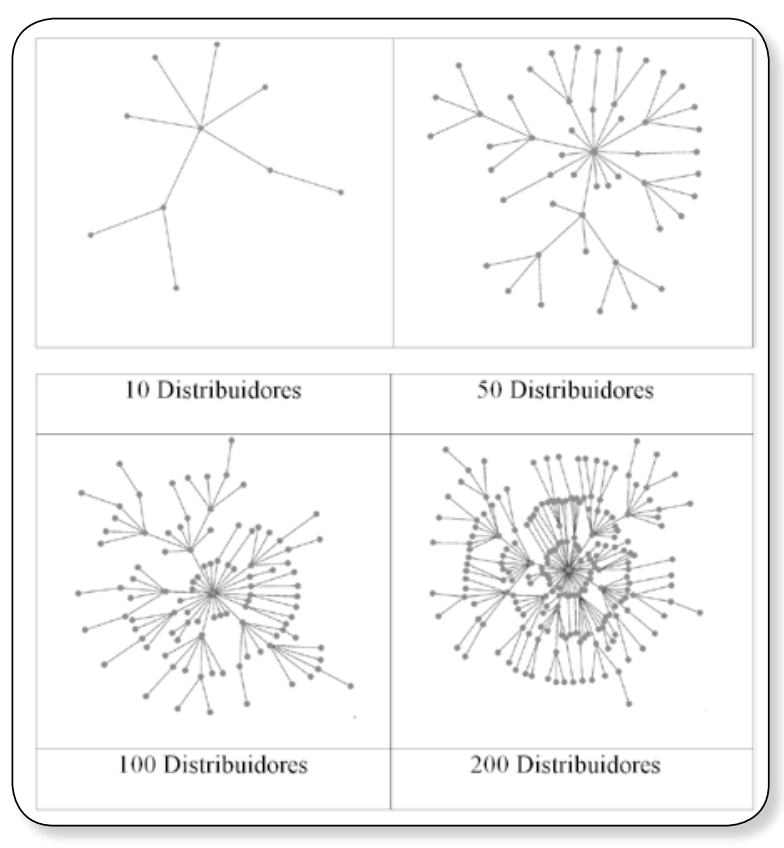

\subsection{Simulación del sistema de pago binario}

Para realizar la simulación del sistema de pago binario primero se escogió una red de mercadeo internacional que tiene operaciones 
en Colombia y se identificaron las principales características de su plan de compensación, como se muestra a continuación:

- Cada persona que ingresa al plan de compensación binario es porque adquiere al menos un producto.

- La comisión por vender un producto es del $10 \%$.

- Siempre hay dos lados, uno izquierdo y uno derecho.

- El plan de compensación se compone de ciclos y cada ciclo tiene 3 pasos, como se muestra en la Figura 1.

- Existen dos seguros para poder cobrar: estar compensado y patrocinar otra nueva persona mínimo cada 3 meses para estar activo.

- Para que un distribuidor pueda cobrar comisiones debe estar compensado en sus dos lados. Si esto no ocurre, cuando se genere el cobro pierde los productos que no cobró en el lado fuerte.

- Para que se realice el primer pago, el distribuidor debe tener al menos una venta dentro de la red.

- El sistema de pago binario pone dos condiciones: la cantidad de productos en el lado débil y el total de productos.

- En la Figura 4, se muestra la explicación de los pasos que conforman un ciclo. La primera condición para que se realice el primer pago es que tenga un producto en el lado débil y mínimo 2 en el lado fuerte. Para que se realice el pago en el segundo paso, el lado débil debe tener mínimo 3 productos y la suma total, mínimo 8 . Para el pago del tercer paso, la suma total debe ser de 15 productos, siempre y cuando el lado débil tenga mínimo 5 productos.
Figura 4. Pasos que conforman un ciclo

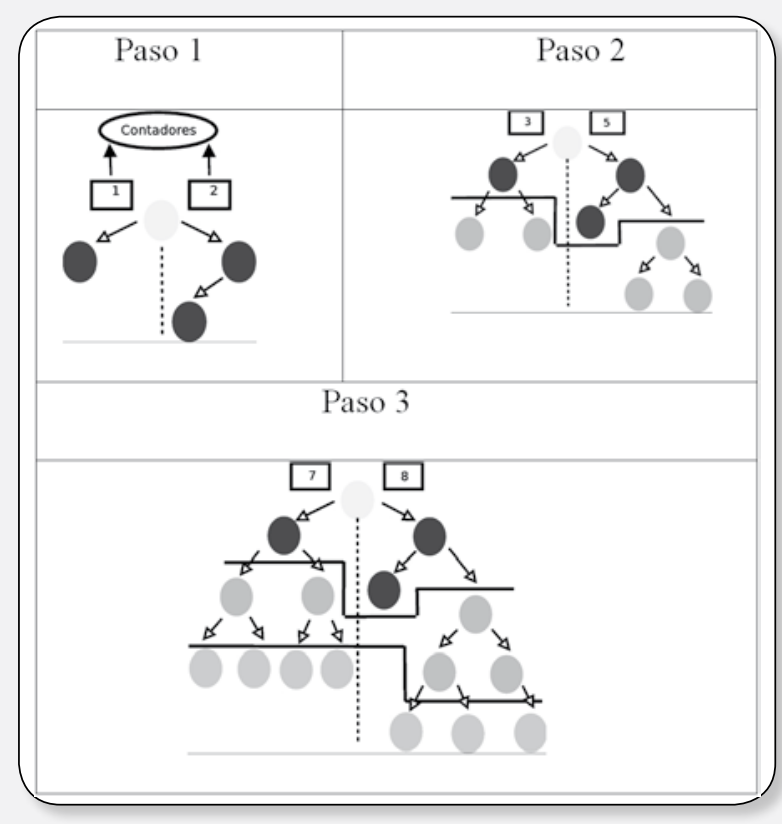

Con la construcción de la red multinivel de 5.000 nodos se puede generar, de acuerdo a los parámetros anteriormente mencionados, una red de compensación binaria y con esto establecer los ingresos de los distribuidores. En la Figura 5 se muestra el diagrama de flujo del algoritmo que fue implementado en Matlab. Para efectos de ilustración, en la Figura 6 se puede observar la red binaria para 100 nodos.

Figura 5. Diagrama de flujo del algoritmo para el sistema de pago binario

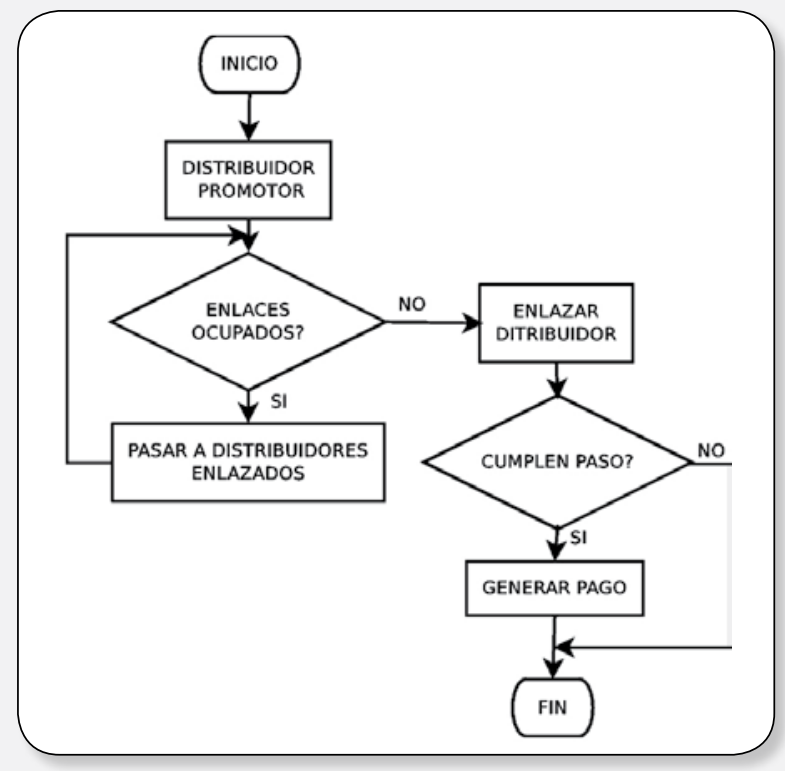




\section{Respuestas}

Año 17

No. 2

Diciembre 2012 ISSN 0122-820X
Se tuvieron en cuenta los siguientes parámetros para realizar el algoritmo del sistema de pago binario en Matlab:

- La comisión por vender un producto es de $\$ 100$.

- Siempre los nodos nuevos entran en las ramas externas del patrocinador.

- Para ubicar el nuevo distribuidor se tuvo en cuenta el lado más débil de su patrocinador, pero si los dos lados tenían igual cantidad de productos, siempre se selecciona el lado izquierdo.

- Para la simulación no se tuvo en cuenta la inactivación de sus distribuidores, ya que para el análisis de los ingresos pasivos los distribuidores deberán estar activos.

Figura 6. Red binaria de 100 distribuidores

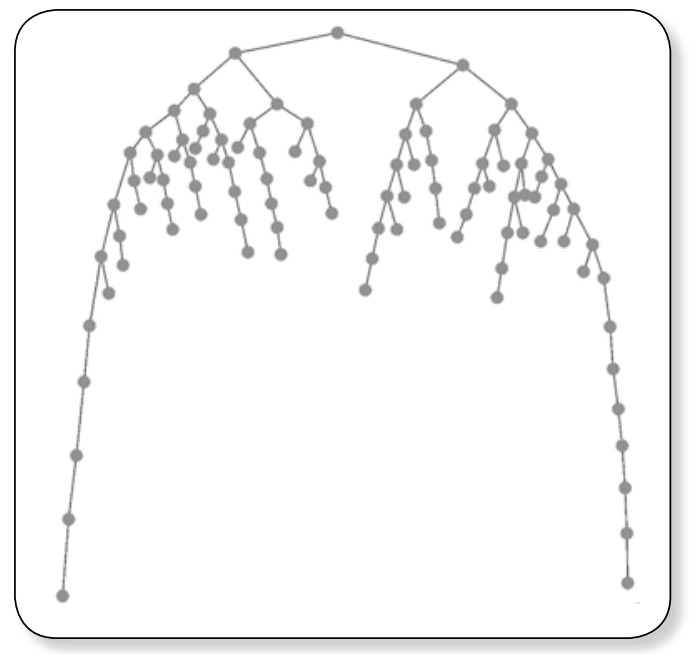

\section{Resultados}

De acuerdo a la red multinivel generada y a las condiciones del sistema de pago implementado, el resultado fue que el $4 \%$ de los distribuidores ganaban el $80 \%$ de las comisiones y el $96 \%$ ganaban el $20 \%$ de las comisiones restantes como se muestra en la Tabla 1. En otras palabras, un porcentaje muy pequeño obtiene la mayoría de los ingresos y la mayoría de distribuidores reciben muy pocos ingresos por comisiones.
Tabla 1. Porcentaje de distribuidores que más ingresos generan.

\begin{tabular}{|c|c|}
\hline Distribuidores & Comisiones \\
\hline $96 \%$ & $20 \%$ \\
\hline $4 \%$ & $80 \%$ \\
\hline
\end{tabular}

Para validar que los ingresos del distribuidor no fueran, en su mayoría, por ventas directas se estableció la siguiente relación (solo para el 4\% de los distribuidores que ganan el $80 \%$ de las comisiones): cantidad de productos vendidos sobre la cantidad de productos cobrados. Los resultados fueron que el distribuidor que más ingresos recibió fue del $3,97 \%$ y para el distribuidor que menos ingresos generó fue de $0,12 \%$. Para tener la certeza que este $4 \%$ de distribuidores hubieran generado ingresos constantes se determinó la cantidad de pagos recibidos, oscilando entre 5 y 947 cheques lo que representa una diferencia muy grande, por lo que se procedió a determinar qué porcentaje de distribuidores recibían el $80 \%$ de los cheques y dio como resultado que el $30 \%$ de los distribuidores generan el $80 \%$ de los cheques, como se muestra en la Tabla 2. Por lo tanto, sólo el 1,2\% del total de distribuidores dentro de la red de 5.000 nodos generaron un ingreso pasivo.

Tabla 2. Porcentaje de cheques generados por el $4 \%$ de distribuidores que más ganan.

\begin{tabular}{|c|c|}
\hline Distribuidores & Cheques \\
\hline $30 \%$ & $80 \%$ \\
\hline $70 \%$ & $20 \%$ \\
\hline
\end{tabular}

\section{Conclusiones}

De la simulación se pudo observar un fenómeno interesante de este tipo de negocios, en cuanto a la forma en que incentiva el trabajo en equipo para generar comisiones. 
El $4 \%$ de los distribuidores, que generaban el $80 \%$ de las comisiones, lo hacían con ventas directas menores al $4 \%$, lo que da a entender que es más importante ayudar a otras personas a generar ingresos. Por lo tanto, las empresas de mercadeo multinivel tienen una buena herramienta para comercializar sus productos, pero, se deben enfocar en crear un sistema que ayude a más distribuidores a generar ingresos pasivos, ya que sólo el 1,2\% de distribuidores generaron dicho ingreso.

Con la simulación se pudo determinar que sólo el 1,2\% de los distribuidores generaban un ingreso pasivo, lo que significa que el $98,8 \%$ no lo lograba y si estos hubieran adquirido los productos sólo por la posibilidad de generar ingresos, el porcentaje de frustración sería muy alto. Por otro lado, si hubieran ingresado única o principalmente por adquirir los productos, sería un esquema de comercialización interesante y se podría aplicar en otros estratos de empresas como la micro y la pequeña (O'Donnell and Cummins, 1999).

Comparado con un sistema tradicional de ventas, donde se contrata a una persona y se le da una comisión por cada venta, el mercadeo multinivel tiene una gran ventaja y es el apalancamiento de las comisiones por medio del esfuerzo de otras personas. Como se mostró en la simulación, los distribuidores que cobraban el $80 \%$ de las comisiones lo hacían con ventas directas de máximo el $4 \%$, por lo tanto, vendían 4 de cada 100 productos cobrados. Esto confirma lo que propuso Brodie et al. (2002), que un sistema de comercialización multinivel puede haber evolucionado más allá de un enfoque táctico en la realización de tareas a un enfoque estratégico, en la facilitación de la finalización de los planes a través de otros.

Como trabajos futuros, se plantean realizar estudios con otros sistemas de pago para determinar su grado de penetración en el mercado y determinar los ingresos de los distribuidores versus los de la compañía multinivel. También realizar simulaciones con varios tamaños de red multinivel, para comparar las variaciones en los ingresos de los distribuidores y las ventas totales de la compañía multinivel. Por último, se debería considerar una población finita y el desgaste de cada nodo para complementar el algoritmo de la red multinivel propuesto por Cruz y Olaya (2008).

\section{Referencias Bibliográficas}

Brodie, S., Stanworth, J., \& Wotruba, T. R. (2002). Comparisons of salespeople in multilevel vs. single level direct selling organizations. Journal of Personal Selling and Sales Management, 22(2), 67-75.

Coughlan, A. T. Grayson, K. (1998). Network Marketing organizations: Compensation plans, retail network growth, and profitability. International Journal of Research in Marketing, 15(5):401- 426.

Cruz, J. (2009). Diffusion dynamics through network marketing structures. Tesis de Maestría. Departamento de Ingeniería industrial. Universidad de los Andes. Bogotá, Colombia. 117 p.

Cruz, J., Olaya, C. (2008). A system dynamics model for studying the structure of network marketing organizations. The 2008 International Conference of the System Dynamics Society. Atenas, Grecia. 20-24 de Julio de 2008.

ISSN 0122-820X

Dumitra, D. (2009). Designing the MLM systems recompensation plans. Review of Management \& Economic Engineering, 8(4):193-203.

Kim, B. J., Jun, T., Kim, J.-Y., Choi, M. (2006). Network marketing on a small-world network. Physica A: Statistical Mechanics and its Applications, 360(2):493-504. 
Kiyosaki, R., Sharon, L. (2000). Rich Dad, Poor Dad: What the Rich Teach Their Kids About Money-That the Poor and Middle Class Do Not. Warner Business Books. 198 p.

Legara, E., Monterola, C., Juanico, D., Litongpalima, M., Saloma, C. (2008). Earning potential in multilevel marketing enterprises. Physica A: Statistical Mechanics and its Applications, 387 (19- 20):4889-4895.

Martinez, M. (2007). Four networks marketings lies that can kill your business. Home Business Magazine, 173(4):54-57.

O'Donnell, A., \& Cummins, D. (1999). The use of qualitative methods to research networking in SMEs. Qualitative Market Research: An International Journal, 2(2), 82-91.

Pedrood, N., Ahmadi, H., and Charafeddine, H. A. (2008). Systematic profitability analysis of binary network marketing organizations. Direct Marketing: An International Journal, 2:81-96.

Taylor, J. (2002). Five causal defining characteristics of and product-based pyramid schemes or recruiting MLM's. Economic Crime Summit Conference 2006. 2002. Revisado en Marzo.

Vander Nat, P. J. and Keep, W.W. (2002). Marketing Fraud: And Approach for Differentiating Multilevel Marketing from Pyramid Schemes. Journal of Public Policy \& Marketing, 21(1):139-151. 